OPEN ACCESS

Edited by:

Zheng Xing,

University of Minnesota Twin Cities,

United States

Reviewed by:

Dong-Yan Jin,

The University of Hong Kong,

Hong Kong

Tao Wang,

Tianjin University, China

Tetsuro Ikegami,

University of Texas Medical Branch at

Galveston, United States

${ }^{*}$ Correspondence:

Xue-jie Yu

yuxuejie@whu.edu.cn

Specialty section: This article was submitted to Viral Immunology,

a section of the journal

Frontiers in Immunology

Received: 06 March 2021 Accepted: 10 May 2021

Published: 27 May 2021

Citation:

Zhou C-m and Yu X-j (2021)

Unraveling the Underlying

Interaction Mechanism Between

Dabie bandavirus and Innate

Immune Response.

Front. Immunol. 12:676861.

doi: 10.3389/fimmu.2021.676861

\section{Unraveling the Underlying Interaction Mechanism Between Dabie bandavirus and Innate Immune Response}

\author{
Chuan-min Zhou ${ }^{1,2}$ and Xue-jie Yu ${ }^{1 *}$ \\ 1 State Key Laboratory of Virology, School of Health Sciences, Wuhan University, Wuhan, China, ${ }^{2}$ Department of Infectious \\ Diseases, Zhongnan Hospital of Wuhan University, Wuhan, China
}

The genus Bandavirus consists of seven tick-borne bunyaviruses, among which four are known to infect humans. Dabie bandavirus, severe fever with thrombocytopenia syndrome virus (SFTSV), poses serious threats to public health worldwide. SFTSV is a tick-borne virus mainly reported in China, South Korea, and Japan with a mortality rate of up to $30 \%$. To date, most immunology-related studies focused on the antagonistic role of SFTSV non-structural protein (NSs) in sequestering RIG-I-like-receptors (RLRs)-mediated type I interferon (IFN) induction and type I IFN mediated signaling pathway. It is still elusive whether the interaction of SFTSV and other conserved innate immune responses exists. As of now, no specific vaccines or therapeutics are approved for SFTSV prevention or treatments respectively, in part due to a lack of comprehensive understanding of the molecular interactions occurring between SFTSV and hosts. Hence, it is necessary to fully understand the host-virus interactions including antiviral responses and viral evasion mechanisms. In this review, we highlight the recent progress in understanding the pathogenesis of SFTS and speculate underlying novel mechanisms in response to SFTSV infection.

Keywords: Bandavirus, immune escape, type I interferon, SFTSV, PRRs

\section{INTRODUCTION}

According to the International Committee on Taxonomy of Viruses (ICTV) (1), the genus Bandavirus, classified under the order Bunyavirales, family Phenuiviridae, comprises 7 species, including Dabie bandavirus, Bhanja bandavirus, Guertu bandavirus, Heartland bandavirus, Hunter Island bandavirus, Kismaayo bandavirus, and Lone Star bandavirus, which are represented by Dabie bandavirus (SFTSV), Bhanja virus (BHAV), Guertu virus (GTV), Heartland virus (HRTV), Hunter island virus (HUIV), Kismaayo virus (KISV), and Lone star virus (LSV) respectively.

Dabie bandavirus, known as severe fever with thrombocytopenia syndrome virus, is a negativesense single-strand RNA virus that was first discovered in 2009 and mainly reported in China, South Korea, Japan, and most recently in Vietnam, Thailand, and Pakistan (2-7). SFTSV is transmitted through tick-bites (like reported Haemaphysalis longicornis and Dermacentor silvarum) and occasionally from person to person via contacting the blood of SFTS patients directly (8-11). 
Haemaphysalis longicornis was reported in Australia, the Pacific regions, and recently in the United States $(12,13)$, indicating transmission risks of SFTSV worldwide. SFTSV causes an emerging hemorrhagic fever disease, termed severe fever with thrombocytopenia syndrome (SFTS), according to its primary symptoms, including fever, thrombocytopenia, and leukocytopenia. Other clinical symptoms of SFTS include anorexia, fatigue, nausea, abdominal pain, vomiting, malaise, diarrhea (2). In 2018, the World Health Organization (WHO) declared SFTS as a priority disease. Many studies investigated the therapeutic or prevention strategies of SFTSV with optimistic results, including vaccine, neutralizing antibody, and antiviral agent (14-19).

Like other bandaviruses, SFTSV has a tripartite RNA genome consisting of Large (L), Medium (M), and Small (S) segments (Figure 1); of which the $\mathrm{L}$ and $\mathrm{M}$ segments are encoded in the negative-sense orientation, while the $\mathrm{S}$ segment is encoded in an ambisense manner. The L segment encodes the RNA-dependent RNA polymerase (RdRp), mediating the replication of the viral genome. The $\mathrm{M}$ segment encodes glycoprotein ( $\mathrm{Gn}$ and $\mathrm{Gc}$ ) precursor that is cleaved into two glycoproteins, Gn and Gc, mediating virion assembly and viral entry into cells. The $S$ segment encodes a non-structural protein (NSs) in the sense orientation and the nucleoprotein (NP) in the antisense orientation, serving as an important virulence factor and mediating genome replication and virion assembly respectively (2).

The innate immune response is the first line of host defense, mainly dependent on pattern recognition receptors (PRRs). Nucleic acids generated from viruses are one of the typical pathogen-associated molecular patterns (PAMPs), which could be specifically targeted by different nucleic acid pattern recognition receptors (PRRs). RIG-I-like-receptors (RLRs) are important in sensing RNA virus infections via recognition of viral
RNA directly, such as eponymous retinoic acid-inducible gene 1 (RIG-I), mainly sensing short ( $<300$ base pairs) dsRNA with a $5^{\prime}$ triphosphate (5' ppp), which can be formed during SFTSV infection (20,21). Activated RIG-I recruits mitochondria outer membrane protein MAVS and mediates downstream signaling cascade activation, leading to the phosphorylation and nuclear translocation of interferon-regulatory factors (IRFs) and nuclear factor kappa-light-chain-enhancer of activated B cells (NF- $\kappa \mathrm{B})$ and triggering the production of type I interferon (IFN). Type I IFNs bind to interferon- $\alpha / \beta$ receptor (IFNAR) mediating the activation of STAT1 and STAT2 heterodimers and resulting in transcriptional activation of IFN-stimulated genes (ISGs), which are crucial arms in the host defense system against viral challenges (22). Accordingly, SFTSV NSs can form inclusion bodies (IBs, also known as viroplasm-like structures) in SFTSV infected and NSs transfected cells. IBs colocalized with lipid droplets, and fatty acid biosynthesis was positively involved in the IBs formation and SFTSV replication (23). SFTSV NSs served as virulence molecules by capturing multiple host molecules into IBs, such as RIG-I, TBK1, IKK, IRF3, IRF7, TRIM25, STAT1, and STAT2 (21, 2427 , which were systematically introduced and discussed in several literatures $(28,29)$. Here, we summarize the current knowledge of innate responses and make hypotheses for the host-virus interaction mechanisms under SFTSV infection status.

\section{AUTOPHAGY IN SFTSV REPLICATION}

Autophagy is an evolutionarily conserved catabolic process that is essential for maintaining cellular homeostasis. In general, autophagic pathways consist of three main routes: microautophagy, chaperone-mediated autophagy (CMA), and macroautophagy (hereafter referred to as autophagy).
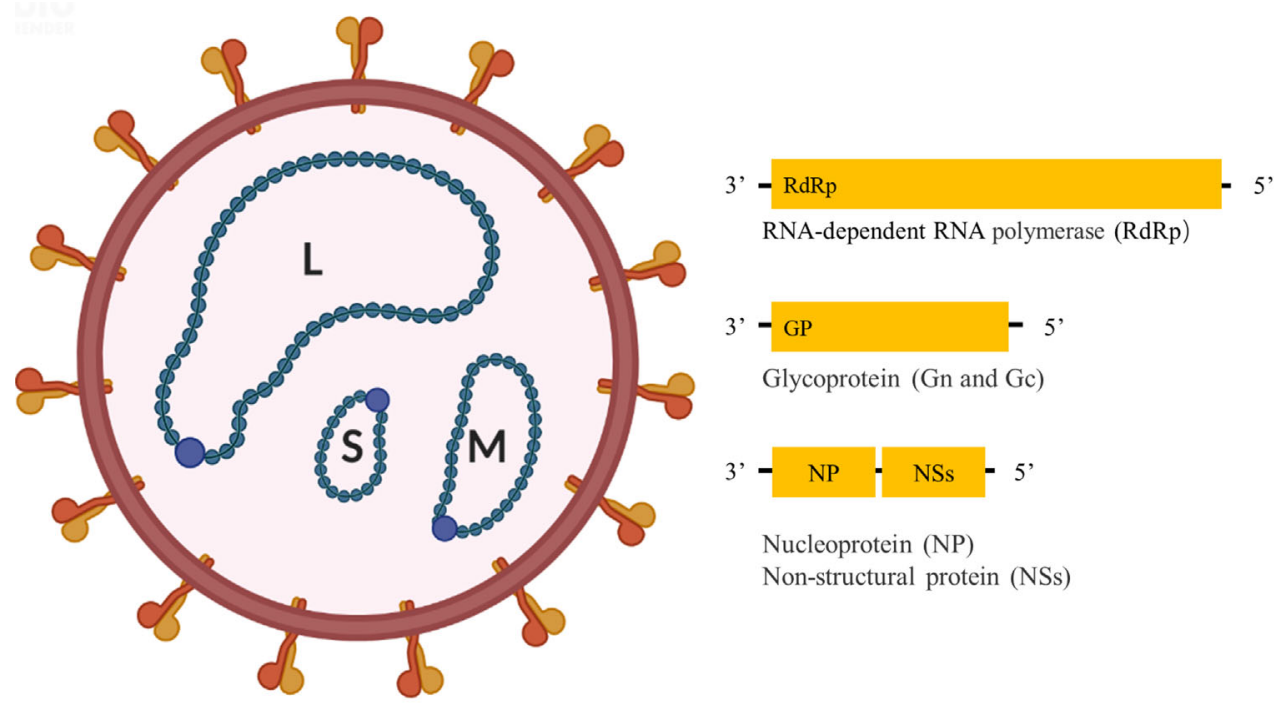

FIGURE 1 | Schematic diagram of SFTSV structure. 
Of which, microautophagy is involved in taking up cytosolic material directly via lysosome membrane invagination, CMA refers to take up substrate proteins with KFERQ-motif selectively via lysosome-associated membrane protein type 2A (LAMP-2A), while autophagy is the best characterized one, involved in engulfing cellular materials into double-membrane vesicles for lysosome degradation. To date, multiple studies have clarified the complicated interconnections of the viral life cycle and the autophagic process $(30,31)$. Typically, autophagy is considered as an anti-viral response by degrading viral particles, enhancing viral antigen presentation, or involved in inflammatory and noninflammatory response modulation. However, autophagy can be sometimes subverted or hijacked at different stages by certain viruses for viral assemble, replication, or even exocytosis.

To date, it is still poorly understood how Bunyavirales affected the host autophagy process and the role of autophagy in Bunyavirales infection. Canonical TLRs are reported to participate autophagy process in macrophages. TLR7 but not other TLRs might interact with and recognize RVFV glycoprotein directly (32). RVFV infection induced autophagy flux via the TLR7-MyD88 axis (33). Inhibition of multiple autophagy stages, from autophagic preinitiation to elongation phase, blocked RVFV infection, revealing a critical role for canonical autophagy in anti-RVFV response. Another study indicated that the Hantaan virus (HTNV), which belongs to the genus Orthohantavirus, induced mitophagy at the early infection stage but incomplete autophagy flux at the late infection stage for viral benefit (34). Autophagy is complexed and different viral proteins might exert reversed or synergic roles on the autophagic process $(35,36)$. In the early stage of HNTV infection, mitophagy was induced by HTNV Gn via interacting with mitochondrial protein TUFM, which inhibited MAVS-associated IFN response via degrading MAVS and therefore provided beneficial conditions for early-stage of HNTV replication. In the late stage of HNTV infection, the induction of autophagy was reversed by NP via disturbing Gn-LC3 interaction and interacting with SNAP29 for preventing autophagosome induction and autophagosome-lysosome formation respectively, which promoted viral replication and exocytosis.

As for SFTSV, limited studies investigated the underlying interaction of SFTSV and autophagy. SFTSV infection promoted the conversion of LC3-I to LC3-II in Vero cells, while lysosomal proteases inhibitor E64d and pepstatin A treatment did not affect the accumulation of LC3-II (37). LC3 deficiency was then found essentially for restricting SFTSV replication. Hence, based on the current information, SFTSV might restrict the autophagic degradation phase for viral replication. An alternative underlying mechanism is that SFTSV might be capable of inducing the occurrence of autophagy but blocking the autophagy flux. However, the amount and quality of data of SFTSV and autophagy was limited and not overwhelming (37), more autophagy-related experiments will be necessary to fully solidify the interaction of autophagy and SFTSV infection, such as detecting adaptor protein p62 and autophagy process by transmission electron microscopy (TEM).

SFTSV NSs exhibited colocalization with LC3, p62, and Lamp2b under SFTSV infection status, while SFTSV NSs alone was incapable of colocalizing with LC3, p62, and Lamp2b under NSs transfection status (37), indicating that other SFTSV proteins, such as RdRp, Gn, Gc, or NP, or host molecules may mediate this binding process. Besides, deletion of atg7 cannot block the colocalization of SFTSV NSs and LC3, indicating that SFTSV NSs induced IBs were not conventional autophagosomes (38). It was worth mentioning that a portion of SFTSV NP was detected in the insoluble fraction and SFTSV NP was then shown interacting with SFTSV NSs protein directly (23), indicating that SFTSV NP but not NSs may be involved in the autophagy process under SFTSV infection, which will be worth for further investigation.

\section{UNFOLDED PROTEIN RESPONSE AND MITOCHONDRION STATUS DURING SFTSV INFECTION}

Unfolded protein response (UPR) is an evolutionarily conserved signaling pathway, induced by ER stress, which plays a critical role in sensing and removing the accumulation of misfolded proteins and restoring homeostasis $(39,40)$. The UPR is mediated by three ER lumen transmembrane proteins and divided into three signaling branches, namely activating transcription factor 6 (ATF6), protein kinase (PKR)-like ER kinase (PERK), and inositol-requiring enzyme 1 (IRE1). Under a resting state, those transmembrane proteins are associated with glucose-regulated protein 78 (BiP). Under ER stress status, increased unfolded or misfolded proteins in the ER lumen promote the dissociation of $\mathrm{BiP}$ from those transmembrane proteins to elicit the UPR process. Like autophagy, UPR plays a double-edged role in response to various virus infections, either promoting virus elimination or subverted for virus invasion, indicating the intimate interaction between host UPR and viral life cycle.

To date, the detailed interactions between bunyaviruses and UPR have not been largely investigated. A recent study indicated that SFTSV infection could activate all three branches of UPR, which also occurred under HRTV and GTV infections. However, only PERK and ATF6, but not IRE1 were involved in favoring SFTSV replication. Further study indicated that GP (Figure 2), but not other viral proteins (RdRp, NP, or NSs) of SFTSV, served as the inducer of the UPR (41). GP localizes to the endoplasmic reticulum-Golgi intermediate compartment (ERGIC) and Golgi complex (42). The assembly and exocytosis process of SFTSV virions occurs via Golgi apparatus and Golgi-derived vesicles respectively $(43,44)$. Hence, activation of the UPR may be induced by the accumulation of unfolded or misfolded proteins in the ER. SFTSV GP can be cleaved into Gn and Gc two subunits. However, it remains elusive whether Gn or Gc play any role in the UPR activation process.

In recent years, many studies have revealed that UPR pathways are connected to the autophagy activation process and many UPRrelated transcription factors participate in autophagy activation by regulating the expression of autophagy-related genes (45). 


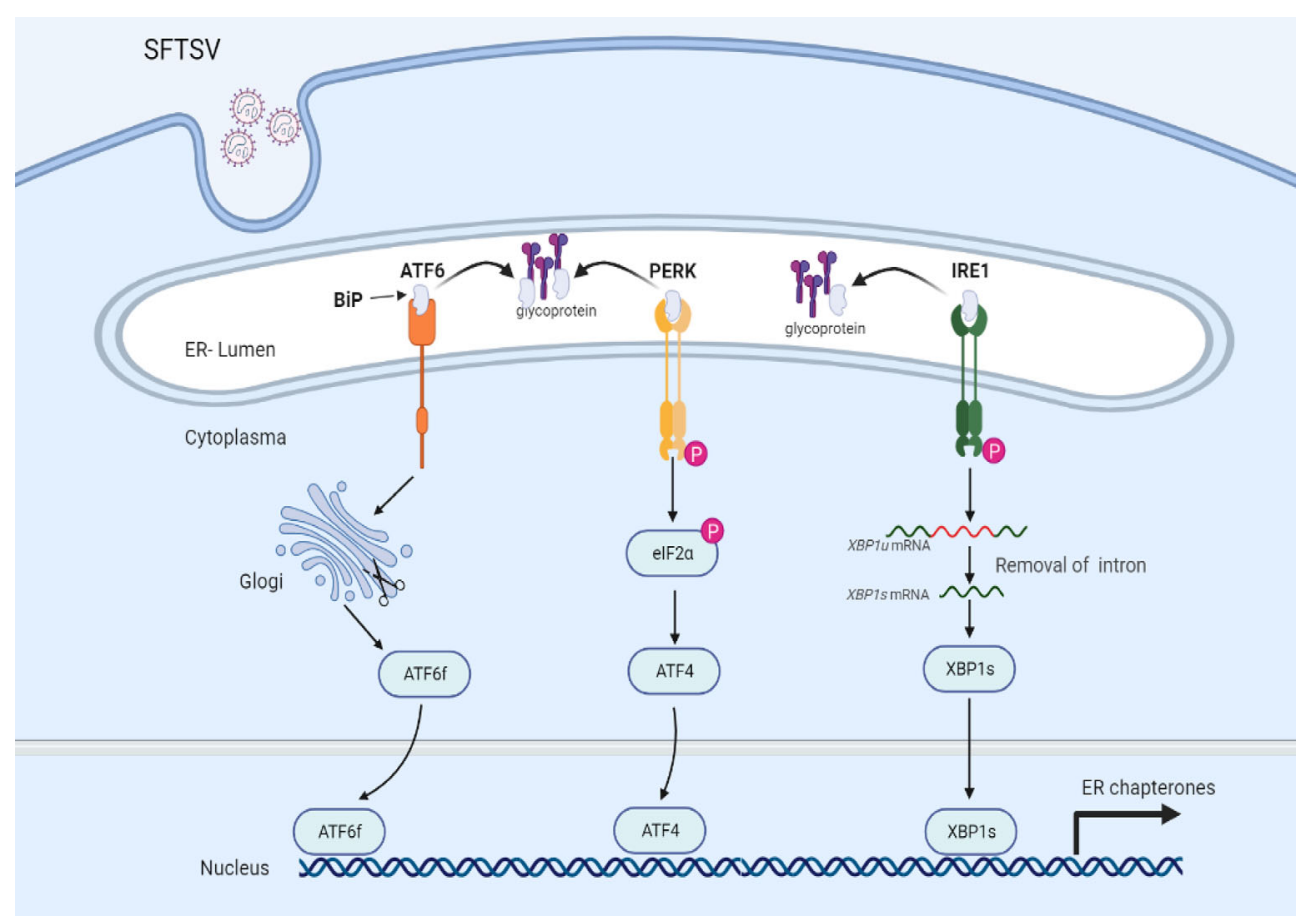

FIGURE 2 | UPR is mediated via SFTSV infection. Under a resting state, transmembrane proteins (ATF6, PERK, and IRE1) are bound with glucose-regulated protein 78 (BiP) maintaining inactive status. Upon SFTSV infection, SFTSV GP mediated the disassociation of BiP with transmembrane proteins, leading to the activation of UPR.

Despite no evidence indicating the interaction of SFTSV-GP and UPR, it is reasonable to propose the interaction between UPR pathways and the autophagic responses under SFTSV infection or SFTSV GP overexpression status. However, the role of UPR and autophagy in modulating SFTSV replication appears controversial. SFTSV replication induced by UPR might be independent of autophagy under SFTSV infection $(37,41)$. Overexpression of HTNV Gn was capable of promoting mitophagy, while NP inhibited Gn-mediated autophagy flux by competing with $\mathrm{Gn}$ for binding to $\operatorname{LC} 3(34,46)$. Hence, it will be interesting to elucidate whether SFTSV- and GP-induced autophagy is associated with UPR.

UPR branches are well known for interacting with inflammatory mediators and triggering the downstream inflammatory pathways through various mechanisms. The complicated interaction between UPR and inflammation is relevant with multiple diseases, such as inflammatory bowel disease, cancer, and neurodegenerative diseases, which is systematically introduced in a recently published literature (47). Previous studies indicated that cytokine storm is associated with SFTS (48). Importantly, apart from IFNs, inflammatory cytokines are essential for innate immune response to some content against virus infection. However, dysregulated or excessive inflammation is harmful and associated with fatal consequences. Hence, it is worth to investigate whether SFTSV-induced inflammation is relevant to UPR.
Mitochondria are ubiquitous cellular organelles, orchestrating multiple biological processes ranging from metabolism to programmed cell death (PCD) (49). Compared with DNA in the nucleus, the cell has a limited capacity to repair mitochondrial DNA (mtDNA). It has been reported for the first time that mtDNA can induce inflammation and arthritis in 2004 (50). To date, many studies have confirmed that mitochondria are critical in mediating host defense against virus infection (51-54). Mitochondria and mtDNA could be transferred to neighboring cells via connexin 43 gap junctions, tunnelling nanotubes, or exosomes (55-57). Under mitochondrion stress status, the mitochondrion is typically combined with ROS accumulation and release of mitochondrial components into the cytoplasm, such as cytochrome $\mathrm{C}$ and mtDNA, which is dependent on the proapoptotic BAK-BAX axis induced inner membrane herniation and mitochondrial outer membrane permeabilization (58). mtDNA is a circular dsDNA molecular harboring unmethylated $\mathrm{CpG}$ motifs similar to bacterial DNA, which mainly exists in the mitochondrial matrix. mtDNA is now accepted as a stimulator of the host immune system (Figure 3).

Remarkably, mitochondrial genome transcription produces mitochondrial dsRNA (mtdsRNA), which can be degraded by mtdsRNA enzyme complexes RNA helicase SUV3 and polynucleotide phosphorylase (PNPase). Recently, mtdsRNA was found serving as a novel DAMP, initiating type I IFN response via classical dsRNA sensor melanoma differentiation- 
associated gene 5 (MDA5) (Figure 3). SUV3 and PNPase deficiency were found to dampen the degradation of mtdsRNA (59). Also, BAK-BAX axis-induced macropores were important for mtdsRNA translocation from mitochondria into the cytoplasm (59). p53 deficiency promoted the accumulation of mtdsRNA in the cytoplasm, which can be recognized by RIG-I after cleaved by RNase L (60).

SFTSV infection disturbed mitochondrial homeostasis, induced mitochondrial damage, and promoted mitochondrial DNA oxidization and cytosolic release (61). We may ask whether mtdsRNA homeostasis is also disturbed under the SFTSV challenge. Previous studies indicated that RIG-I and MDA-5 were both upregulated under SFTSV infection (62, 63). RIG-I was involved in the recognition of SFTSV infection by sensing SFTSV RNA directly, while MDA5 was mainly sensing long dsRNA. Hence, it is interesting to explore the underlying mechanism of whether SFTSV infection would promote mtdsRNA accumulation and activate RIG-I and MDA5 dependent antiviral response.

Apart from those classical cytosolic RNA sensors like RIG-I and MDA5, Toll-like receptors (TLRs), including RNA sensor TLR3, TLR7, and TLR8, are located in endosomes, of which TLR3 senses dsRNA, whereas TLR7 and TLR8 are activated by ssRNA. Activated TLR3 mediates the activation of IRF3 and NF- $\mathrm{KB}$ via TRIF, while TLR7 and TLR8 utilize the adaptor MyD88 to activate

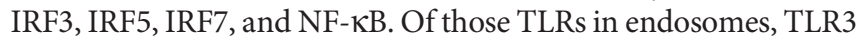
was recently found capable of recognizing alcohol-associated stress-induced mtdsRNA accumulation (64). TLR3 expression was upregulated significantly compared with TLR-7 and TLR-8 under SFTSV infection in HepG2 cells (63). However, another study found that SFTSV suppressed the expression of TLR3 in both monocytes and myeloid dendritic cells (65). Hence, it is interesting to investigate the detailed role of TLR3 in different cell types responding to the SFTSV infection.

\section{PYROPTOSIS IN ANTI-SFTSV IMMUNITY}

Previous studies showed that SFTSV patients exhibited elevated levels of pro-inflammatory cytokines $(48,66)$, such as interleukin$1 \beta$ (IL-1 1 ), and cytokine storms could serve as biomarkers of SFTS patients. It should be noted that pro-inflammatory cytokines constitute a double-edged sword. On one hand, proper proinflammatory cytokines are key mediators of the innate immune system, playing an important role in host defense against virus attack. On the other hand, severe cytokine storms cause severe tissue damage in patients. In the context of the normal innate immune system, a well-coordinated and rapid innate immune response is the first line of defense against viral infection. Among those known PRRs, inflammasomes, including NLRs (NLRP3, and NLRC4) and ALRs (AIM2 and IFI16), play an important role in modulating the inflammatory response and cell pyroptosis. Typically, inflammasomes are a group of multi-protein platforms, containing inflammasome-initiating sensors, adaptor protein ASC, and pro-caspase-1, which are important for recognizing cellular damage- or pathogens-derived signals and orchestrating host

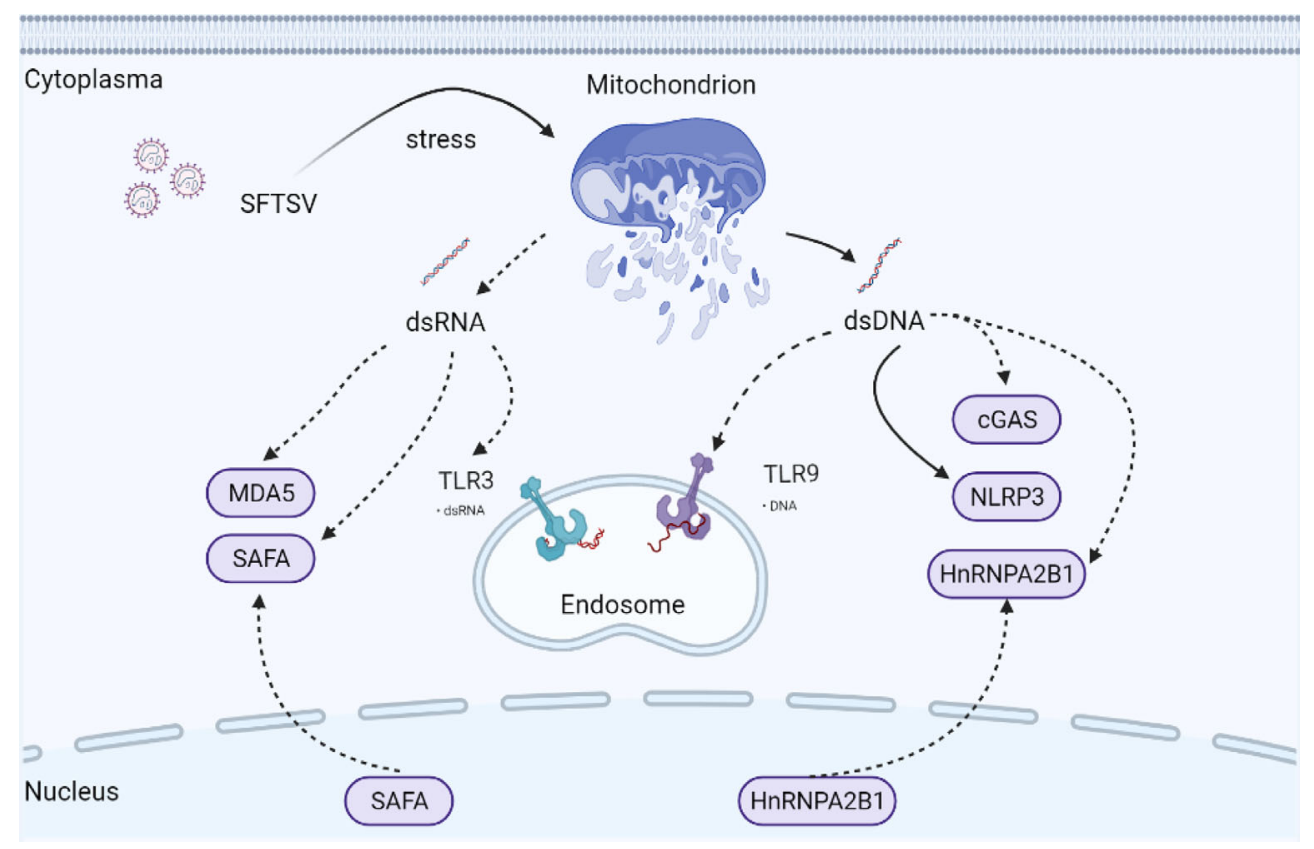

FIGURE 3 | Possible underlying interaction of mitochondria and PRRs under SFTSV infection. Mitochondria are ubiquitous cellular organelles, orchestrating multiple biological processes. Under mitochondrion stress status, BAX/BAK axis is activated mediating mitochondrial permeabilization. mtdsDNA and mtdsRNA are then released into the cytoplasm, which triggers the activation of cytosolic PRRs mediated innate immune response. Solid arrows indicate confirmed mechanisms under SFTSV infection. Dotted arrows indicate unconfirmed or proposed mechanisms under SFTSV infection. 
defense against pathogen invasion (67). It is worth mentioning that NLRP3 inflammasome is the best-characterized inflammasome and to be associated with inflammation under RNA virus infection (68). NLRP3 inflammasome activation is divided into priming and maturation processes. Briefly, the TLR-NF- $\kappa B$ axis enhances the transcription of pro-Il-1 $\beta$ and inflammasome-associated components. Activated NLRP3 protein then recruits adaptor protein ASC and pro-caspase-1, inducing the pro-caspase-1 autoproteolytic cleavage into the active form caspase-1, promoting the maturation and secretion of pro-inflammatory cytokines IL-1 $\beta$ and IL-18 and driving gasdermin D (GSDMD)-dependent pyroptosis (69).

NLRP3 recognizes a variety of PAMPs and DAMPs, such as RNA, ATP, ionic flux, and lysosomal damage $(69,70)$. Importantly, mitochondria have diverse functions, ranging from energy conversion to maintaining cellular homeostasis. Accumulating data have indicated that mitochondria are central regulators triggering NLRP3 inflammasome-dependent antiviral immune responses and facilitating viral eradication (69, 71, 72). Mitochondria promote the NLRP3 inflammasome assembly via MAVS/Mfn2/MAM axis. The destabilization of mitochondria is believed to inducing NLRP3 inflammasome activation via ROS, $\mathrm{Ca}^{2+}, \mathrm{NAD}^{+}$, or mtDNA. Additionally, autophagy-mediated clearance of damaged mitochondria is confirmed prohibiting the activation of NLRP3 inflammasome (73).

Recent studies indicated strong NF- $\kappa B$ activation under SFTSV infection $(61,63,74)$. The priming stage of inflammasome activation, including the transcription of $I L-1 \beta$, is dependent on NF- $\kappa \mathrm{B}$ activation. SFTSV infection activated BAX/BAK axis, reduced mitochondrial membrane potential, and induced mtROS production, strongly indicating that SFTSV infection caused dysfunction and damage of mitochondria (61). Generally, mitochondrial dysfunction is often associated with oxidization and the release of mtDNA into the cytosol. BAX/BAK mediatedpermeabilization on the mitochondrial membrane is essential for the mitochondria-cytoplasm translocation of mtDNA (58). SFTSV infection was confirmed facilitating the maturation and secretion of pro-inflammatory cytokine IL-1 $\beta$ through NLRP3 inflammasome (61). NLRP3 mediated inflammatory response was associated with SFTSV fatal infection outcome. However, it remains elusive whether other inflammasomes are involved in recognizing SFTSV infection.

Similarly, the NLRP3 inflammasome is capable of recognizing RVFV and HNTV infection $(75,76)$. RVFV alone could barely promote the activation of the NLRP3 inflammasome. LPSinduced priming process was necessary for enhancing the activation of NLRP3 inflammasome under RVFV challenge, but not UV-RVFV. Unlike SFTSV, RVFV NSs was capable of forming filamentous structure in the nucleus, which was reported inducing transcriptional inhibition via interrupting of TFIIH complex assembly or promoting the degradation of TFIIH p62 subunit $(77,78)$. Enhanced production of IL-1 $\beta$ was expected in RVFV NSs deficient strain, indicating that RVFV NSs may be involved in immune escape of NLRP3 inflammasome (76). The priming stage of the inflammasome might be disturbed by RVFV NSs. Besides, both SFTSV NSs and HRTV NSs suppressed NLRP3 inflammasome-dependent IL-1 $\beta$ secretion (79). However, NSs of SFTSV promoted the activation of NF- $\kappa B(63,74)$. Despite that NSs of SFTSV, HRTV, and RVFV were shown to restrict NLRP3-mediated antiviral immune responses, the detailed mechanism is still elusive. Many questions remain to be addressed. What is the conserved domain of NSs in restricting NLRP3 inflammasome activation? What are the NSs targeted inflammasome-related proteins?

\section{POSSIBLE INTERACTION OF SFTSV WITH NOVEL NUCLEIC ACID SENSORS}

Apart from NLRP3, mtDNA interacts closely with multiple additional PRRs, including cyclic GMP-AMP synthase (cGAS), TLR9, NLRC4, and AIM2 $(80,81)$. cGAS is a well-characterized cytosolic DNA sensor catalyzing the generation of the nucleotide second messenger cyclic AMP-GMP (cGAMP), which then activates the stimulator of interferon gene (STING) $(82,83)$. Activated STING translocates from ER to ERGIC and Golgi, activating TBK1-IRF3 dependent IFN and proinflammatory responses (84), serving as the major defense mechanism against microbial infection. Several studies have indicated that cGAS is also responsible for sensing RNA virus challenges indirectly by recognizing mtDNA $(51,54)$. However, it is still elusive whether and how cGAS was activated by SFTSV. Now that mtDNA oxidization and cytosolic release were induced under SFTSV infection (61), it is reasonable to deduce that the cGAS-mediated IFN signal pathway could be activated by SFTSV-induced mtDNA release.

Apart from cellular viruses, many DNA viruses direct viral DNA into the nucleus. Accordingly, DNA sensors are also capable of existing and taking function in the nucleus, such as cGAS and IFI16 $(85,86)$. In 2019, Cao and coworkers confirmed a novel nuclear DNA sensor, namely heterogeneous nuclear ribonucleoprotein A2B1 (hnRNPA2B1), which is previously confirmed participating in RNA regulatory network (87-89). hnRNPA2B1 was activated and translocated from nucleus to cytoplasm, where it mediated cGAS-independent but STINGdependent innate immune response to DNA virus (90). It is well established that STING can be activated by bacterial cyclic dinucleotides (CDNs), such as cyclic di-AMP (c-di-AMP), as well as cGAMP. Phosphorylation of STING at $\operatorname{Ser}^{366}$ and Leu ${ }^{374}$ is important for the activation of STING $(91,92)$. However, it remains elusive how hnRNPA2B1 activates STING. Is hnRNPA2B1 participating in the phosphorylation of STING or promoting the translocating of STING? What is the conserved domain mediating the interaction of hnRNPA2B1 and STING?

hnRNPA2B1 was important for the innate immune-mediated inhibition of DNA virus replication, including HSV-1 and AdV, but not involved in the recognition of RNA virus VSV and SeV. Upon sensing viral DNA, nuclear-localized hnRNPA2B1 formed a complex with viral DNA and then formed a homodimer, which was then demethylated at arginine- 226 by the arginine demethylase JMJD6. Mutation of the hnRNPA2B1 dimer interface was unable to associate with JMJD6 and abrogated hnRNPA2B1 nucleocytoplasmic translocation, inhibiting the subsequent 
initiation of the IFN- $\beta$ signal pathway. Additionally, hnRNPA2B1 facilitated $\mathrm{m}^{6} \mathrm{~A}$ modification and nucleocytoplasmic trafficking of mRNA cGAS, IFI16, and STING to amplify the activation of the IFN- $\beta$ signal pathway in response to DNA virus infection. It remains unknown whether all RNA viruses not capable of activating hnRNPA2B1? Despite that hnRNPA2B1 mainly existed in the nucleus under HSV-1 infection, of interest to us is whether mtDNA could directly promote the activation of cytoplasmic hnRNPA2B1 or enhance the activation of nuclear hnRNPA2B1 under DNA virus infection.

In 2019, SAFA (nuclear scaffold attachment factor A) was identified as a novel nuclear dsRNA sensor (previously known as nuclear matrix protein hnRNPU) for both DNA and RNA viruses. Like hnRNPA2B1, SAFA was also a nucleic acid binding protein belonging to the hnRNP family. Upon sensing viral RNA in the nucleus, nuclear matrix protein SAFA oligomerized and promoted antiviral gene expression (93). Myeloid-specific SAFA-deficient mice showed decreased IFN- $\beta$ activation, which was more susceptible and showed less resistance to HSV-1 and VSV infection. SAFA directly sensed viral RNA in the nucleus via its RGG domain and form homodimers. SAFA interacted with SMARCA5 and TOP1, two key components of the SWI/SNF nucleosome remodeling complex, via the middle SPRY domain leading to the transformation of chromosome conformation and activation of the enhancers and super-enhancers of antiviral genes.

The induction of SAFA by the virus was severely impaired in ELF4, STING, or MAVS deficient cells. Further studies indicated that ELF4 significantly promoted the expression of SAFA and the phenomena were further enhanced by STING and MAVS overexpression, indicating STING and MAVS may promote the expression of SAFA. The authors hypothesized that SAFA may take function through the STING or MAVS-dependent antiviral IFN pathway and was required for IRF3 and IRF7 recruitment to the IFN- $\beta$ promoter. Based on the current information, SAFA took function in a cytoplasm-independent manner under VSV or HSV1 infection, which cannot affect the activation and phosphorylation of IRF3. The detailed function of SAFA under virus infection is largely elusive. It will be an interesting topic to investigate whether SAFA can be translocated to cytoplasm like hnRNPA2B1 under infection status.

\section{ROLE OF APOPTOSIS UNDER SFTSV CHALLENGE}

Apoptosis is a major form of regulated cell death, characterized by nuclear condensation, cell shrinkage, membrane blebbing, and DNA fragmentation $(94,95)$. Apoptosis is initiated through the extrinsic receptor-mediated pathway and intrinsic mitochondrial pathway. The extrinsic pathway is activated by extracellular stimulation via death receptors, such as TNF receptor, Fas, and TRAIL receptor. The intrinsic apoptotic pathway is activated by various intracellular stimuli, such as cytochrome c DNA damage, oxidative stress, and ER stress. Of note, mitochondria are crucial for the activation of apoptosis $(96,97)$. Under cellular stress conditions, the Bcl-2 effector proteins BAK/BAX cause mitochondrial membrane permeabilization, which in turn promotes the release of cytochrome $\mathrm{c}$ into cytoplasm, leading to the activation of apoptosis executioner caspase endopeptidases, caspase- 3 and -7 , cleaving hundreds of cell substrates, and inducing apoptosis. Additionally, upon apoptosis receptor activation, the intracellular receptor region recruits the adapter protein $\mathrm{FADD}$, which in turn mediates the activation of caspases- 3 and -7 induced apoptosis (98). Besides, the immune response induced by $\mathrm{mtDNA}$ leakage is partially prevented by the activation of apoptosis and related caspase activity, including caspase-3, -7, and -9. Mitochondrion-related caspases are shown cleaving or inhibiting the activation of multiple PRRs directly, such as cGAS, STING, IRF3, or MAVS (99-101).

Apoptosis is a highly regulated process, eliciting both negative and positive effects on the viral life cycle. On one hand, apoptosis is involved in eliminating virus-infected cells. On the other hand, viruses, mainly through viral proteins, might take advantage of apoptosis for exocytosis via regulating the expression and function of death receptors, caspase endopeptidases, or apoptotic proteins $(102,103)$. Previous studies indicated that apoptosis was not or slightly induced under SFTSV infection in monocytes $(61,62)$, and this was supported by SFTSV related proteomic analysis, which revealed that several proteins with anti-apoptosis roles were upregulated under SFTSV infection, such as SOD2, BCL3, CD74, FAM129B (61). Apoptosis may be involved in SFTSV-associated immunopathology. However, the detailed mechanisms remain unclear and further study will be necessary to investigate the balance mechanism of apoptosis.

\section{KNOWLEDGE GAP}

Currently, there is no vaccine or treatment available to prevent SFTS. SFTSV poses a serious threat to public health, while the pathogenic mechanism of SFTSV is largely elusive. The viralmediated immune responses are complex processes. It is just a beginning to unravel the detailed interacted mechanisms of SFTSV in host defense. In this review, we have discussed the wellestablished role and highlighted the underlying mechanism of innate immunity in response to SFTSV infection. Several important questions remain to be answered: Are SFTSV viral components (not limited to NSs) taking part in the immune escape of those innate immune responses? How can SFTSV hijack the host process for viral replication? How can autophagy participate in sensing and defending SFTSV challenges? Is the UPR related to SFTSV-induced autophagy and inflammation? What are the detailed mechanisms of SFTSV in inducing mitochondrial stress? Are mitochondria playing a key role in host defense against SFTSV infections? Are mtdsRNA and mtDNA taking part in the modulation of classical RNA and DNA PRRs under the SFTSV challenge? Are the nucleic acid sensors such as SAFA or hnRNPA2B1 capable of sensing SFTSV infection? If so, what is the immune escape mechanism of SFTSV? Answering and discovering those possible interconnections will not only enhance our understanding of the viral mechanism to interact with host immunity but also lead to the potential anti-SFTSV strategies. 


\section{AUTHOR CONTRIBUTIONS}

Conceptualization, C-mZ. Original draft preparation, C-mZ. Writing-review and editing, C-mZ and $\mathrm{X}-\mathrm{jY}$. Project administration, C-mZ and X-jY. Funding acquisition, C-mZ and $\mathrm{X}-\mathrm{jY}$. All authors contributed to the article and approved the submitted version.

\section{REFERENCES}

1. Kuhn JH, Adkins S, Alioto D, Alkhovsky SV, Amarasinghe GK, Anthony SJ, et al. 2020 Taxonomic Update for Phylum Negarnaviricota (Riboviria: Orthornavirae), Including the Large Orders Bunyavirales and Mononegavirales. Arch Virol (2020) 165(12):3023-72. doi: 10.1007/s00705020-04731-2

2. Yu XJ, Liang MF, Zhang SY, Liu Y, Li JD, Sun YL, et al. Fever With Thrombocytopenia Associated With a Novel Bunyavirus in China. New Engl $J$ Med (2011) 364(16):1523-32. doi: 10.1056/NEJMoa1010095

3. Kim KH, Yi J, Kim G, Choi SJ, Jun KI, Kim NH, et al. Severe Fever With Thrombocytopenia Syndrome, South Korea, 2012. Emerging Infect Dis (2013) 19(11):1892-4. doi: 10.3201/eid1911.130792

4. Takahashi T, Maeda K, Suzuki T, Ishido A, Shigeoka T, Tominaga T, et al. The First Identification and Retrospective Study of Severe Fever With Thrombocytopenia Syndrome in Japan. J Infect Dis (2014) 209(6):816-27. doi: 10.1093/infdis/jit603

5. Tran XC, Yun Y, Van An L, Kim SH, Thao NTP, Man PKC, et al. Endemic Severe Fever With Thrombocytopenia Syndrome, Vietnam. Emerging Infect Dis (2019) 25(5):1029-31. doi: 10.3201/eid2505.181463

6. Ongkittikul S, Watanawong R, Rompho P. Severe Fever With Thrombocytopenia Syndrome Virus: The First Case Report in Thailand. BKK Med J (2020) 16(2):204. doi: 10.31524/bkkmedj.2020.22.001

7. Zohaib A, Zhang J, Saqib M, Athar MA, Hussain MH, Chen J, et al. Serologic Evidence of Severe Fever With Thrombocytopenia Syndrome Virus and Related Viruses in Pakistan. Emerging Infect Dis (2020) 26(7):1513-6. doi: 10.3201/eid2607.190611

8. Luo LM, Zhao L, Wen HL, Zhang ZT, Liu JW, Fang LZ, et al. Haemaphysalis Longicornis Ticks as Reservoir and Vector of Severe Fever With Thrombocytopenia Syndrome Virus in China. Emerging Infect Dis (2015) 21(10):1770-6. doi: 10.3201/eid2110.150126

9. Liu Y, Li Q, Hu W, Wu J, Wang Y, Mei L, et al. Person-to-Person Transmission of Severe Fever With Thrombocytopenia Syndrome Virus. Vector Borne Zoonotic Dis (2012) 12(2):156-60. doi: 10.1089/ vbz.2011.0758

10. Hu YY, Zhuang L, Liu K, Sun Y, Dai K, Zhang XA, et al. Role of Three Tick Species in the Maintenance and Transmission of Severe Fever With Thrombocytopenia Syndrome Virus. PloS neglected Trop Dis (2020) 14(6): e0008368. doi: 10.1371/journal.pntd.0008368

11. Bao CJ, Guo XL, Qi X, Hu JL, Zhou MH, Varma JK, et al. A Family Cluster of Infections by a Newly Recognized Bunyavirus in Eastern China, 2007: Further Evidence of Person-to-Person Transmission. Clin Infect Dis an Off Publ Infect Dis Soc America (2011) 53(12):1208-14. doi: 10.1093/cid/cir732

12. Hutcheson HJ, Dergousoff SJ, Lindsay LR. Haemaphysalis Longicornis: A Tick of Considerable Veterinary Importance, Now Established in North America. Can Veterinary J = La Rev veterinaire Can (2019) 60(1):27-8. doi: $10.1172 /$ jci.insight. 136855

13. Raghavan RK, Barker SC, Cobos ME, Barker D, Teo EJM, Foley DH, et al. Potential Spatial Distribution of the Newly Introduced Long-Horned Tick, Haemaphysalis Longicornis in North America. Sci Rep (2019) 9(1):498. doi: 10.1038/s41598-018-37205-2

14. Takayama-Ito M, Saijo M. Antiviral Drugs Against Severe Fever With Thrombocytopenia Syndrome Virus Infection. Front Microbiol (2020) 11:150. doi: $10.3389 /$ fmicb.2020.00150

15. Wu X, Li Y, Huang B, Ma X, Zhu L, Zheng N, et al. A Single-Domain Antibody Inhibits SFTSV and Mitigates Virus-Induced Pathogenesis In Vivo. JCI Insight (2020) 5(13):e136855. doi: 10.1172/jci.insight.136855

\section{FUNDING}

This study was supported by the National Natural Science Funds of China (81971939 and 31570167) and the Fundamental Research Funds for the Central Universities (2042021kf0046). The funders had no role in the study design, data collection and analysis, decision to publish, or the preparation of the manuscript.

16. Kim KH, Kim J, Ko M, Chun JY, Kim H, Kim S, et al. An Anti-Gn Glycoprotein Antibody From a Convalescent Patient Potently Inhibits the Infection of Severe Fever With Thrombocytopenia Syndrome Virus. PloS Pathog (2019) 15(2):e1007375. doi: 10.1371/journal.ppat.1007375

17. Tani H, Fukuma A, Fukushi S, Taniguchi S, Yoshikawa T, Iwata-Yoshikawa $\mathrm{N}$, et al. Efficacy of T-705 (Favipiravir) in the Treatment of Infections With Lethal Severe Fever With Thrombocytopenia Syndrome Virus. mSphere (2016) 1(1):e00061-15. doi: 10.1128/mSphere.00061-15

18. Dong F, Li D, Wen D, Li S, Zhao C, Qi Y, et al. Single Dose of a rVSV-based Vaccine Elicits Complete Protection Against Severe Fever With Thrombocytopenia Syndrome Virus. NPJ Vaccines (2019) 4:5. doi: 10.1038/ s41541-018-0096-y

19. Kwak JE, Kim YI, Park SJ, Yu MA, Kwon HI, Eo S, et al. Development of a SFTSV DNA Vaccine That Confers Complete Protection Against Lethal Infection in Ferrets. Nat Commun (2019) 10(1):3836. doi: 10.1038/s41467-019-11815-4

20. Rehwinkel J, Gack MU. Rig-I-like Receptors: Their Regulation and Roles in RNA Sensing. Nat Rev Immunol (2020) 20(9):537-51. doi: 10.1038/s41577020-0288-3

21. Min YQ, Ning YJ, Wang H, Deng F. A RIG-I-like Receptor Directs Antiviral Responses to a Bunyavirus and Is Antagonized by Virus-Induced Blockade of TRIM25-mediated Ubiquitination. J Biol Chem (2020) 295(28):9691-711. doi: 10.1074/jbc.RA120.013973

22. Ning YJ, Wang M, Deng M, Shen S, Liu W, Cao WC, et al. Viral Suppression of Innate Immunity Via Spatial Isolation of TBK1/IKKepsilon From Mitochondrial Antiviral Platform. J Mol Cell Biol (2014) 6(4):324-37. doi: $10.1093 / \mathrm{jmcb} / \mathrm{mju} 015$

23. Wu X, Qi X, Liang M, Li C, Cardona CJ, Li D, et al. Roles of Viroplasm-Like Structures Formed by Nonstructural Protein NSs in Infection With Severe Fever With Thrombocytopenia Syndrome Virus. FASEB J Off Publ Fed Am Societies Exp Biol (2014) 28(6):2504-16. doi: 10.1096/fj.13-243857

24. Hong Y, Bai M, Qi X, Li C, Liang M, Li D, et al. Suppression of the IFN-alpha and -Beta Induction Through Sequestering IRF7 Into Viral Inclusion Bodies by Nonstructural Protein NSs in Severe Fever With Thrombocytopenia Syndrome Bunyavirus Infection. J Immunol (2019) 202(3):841-56. doi: 10.4049/jimmunol.1800576

25. Yamada S, Shimojima M, Narita R, Tsukamoto Y, Kato H, Saijo M, et al. RigI-Like Receptor and Toll-Like Receptor Signaling Pathways Cause Aberrant Production of Inflammatory Cytokines/Chemokines in a Severe Fever With Thrombocytopenia Syndrome Virus Infection Mouse Model. J Virol (2018) 92(13):e02246-17. doi: 10.1128/JVI.02246-17

26. Rezelj VV, Li P, Chaudhary V, Elliott RM, Jin DY, Brennan B. Differential Antagonism of Human Innate Immune Responses by Tick-Borne Phlebovirus Nonstructural Proteins. mSphere (2017) 2(3):e00234-17. doi: 10.1128/ mSphere.00234-17

27. Chaudhary V, Zhang S, Yuen KS, Li C, Lui PY, Fung SY, et al. Suppression of Type I and Type III IFN Signalling by NSs Protein of Severe Fever With Thrombocytopenia Syndrome Virus Through Inhibition of STAT1 Phosphorylation and Activation. J Gen Virol (2015) 96(11):3204-11. doi: 10.1099/jgv.0.000280

28. Mendoza CA, Ebihara H, Yamaoka S. Immune Modulation and ImmuneMediated Pathogenesis of Emerging Tickborne Banyangviruses. Vaccines (2019) 7(4):125. doi: 10.3390/vaccines7040125

29. Wuerth JD, Weber F. Phleboviruses and the Type I Interferon Response. Viruses (2016) 8(6):174. doi: 10.3390/v8060174

30. Choi Y, Bowman JW, Jung JU. Autophagy During Viral Infection - A Double-Edged Sword. Nat Rev Microbiol (2018) 16(6):341-54. doi: 10.1038/ s41579-018-0003-6 
31. Keller MD, Torres VJ, Cadwell K. Autophagy and Microbial Pathogenesis. Cell Death Differ (2020) 27(3):872-86. doi: 10.1038/s41418-019-0481-8

32. Nakamoto M, Moy RH, Xu J, Bambina S, Yasunaga A, Shelly SS, et al. Virus Recognition by Toll-7 Activates Antiviral Autophagy in Drosophila. Immunity (2012) 36(4):658-67. doi: 10.1016/j.immuni.2012.03.003

33. Moy RH, Gold B, Molleston JM, Schad V, Yanger K, Salzano MV, et al. Antiviral Autophagy Restrictsrift Valley Fever Virus Infection and Is Conserved From Flies to Mammals. Immunity (2014) 40(1):51-65. doi: 10.1016/j.immuni.2013.10.020

34. Wang K, Ma H, Liu H, Ye W, Li Z, Cheng L, et al. The Glycoprotein and Nucleocapsid Protein of Hantaviruses Manipulate Autophagy Flux to Restrain Host Innate Immune Responses. Cell Rep (2019) 27(7):2075-91.e5. doi: 10.1016/j.celrep.2019.04.061

35. Ding B, Zhang G, Yang X, Zhang S, Chen L, Yan Q, et al. Phosphoprotein of Human Parainfluenza Virus Type 3 Blocks Autophagosome-Lysosome Fusion to Increase Virus Production. Cell Host Microbe (2014) 15(5):56477. doi: 10.1016/j.chom.2014.04.004

36. Ding B, Zhang L, Li Z, Zhong Y, Tang Q, Qin Y, et al. The Matrix Protein of Human Parainfluenza Virus Type 3 Induces Mitophagy That Suppresses Interferon Responses. Cell Host Microbe (2017) 21(4):538-47 e4. doi: 10.1016/j.chom.2017.03.004

37. Sun Y, Liu MM, Lei XY, Yu XJ. SFTS Phlebovirus Promotes LC3-II Accumulation and Nonstructural Protein of SFTS Phlebovirus CoLocalizes With Autophagy Proteins. Sci Rep (2018) 8(1):5287. doi: 10.1038/s41598-018-23610-0

38. Santiago FW, Covaleda LM, Sanchez-Aparicio MT, Silvas JA, Diaz-Vizarreta AC, Patel JR, et al. Hijacking of RIG-I Signaling Proteins Into Virus-Induced Cytoplasmic Structures Correlates With the Inhibition of Type I Interferon Responses. J Virol (2014) 88(8):4572-85. doi: 10.1128/JVI.03021-13

39. Bettigole SE, Glimcher LH. Endoplasmic Reticulum Stress in Immunity. Annu Rev Immunol (2015) 33:107-38. doi: 10.1146/annurev-immunol-032414-112116

40. Grootjans J, Kaser A, Kaufman RJ, Blumberg RS. The Unfolded Protein Response in Immunity and Inflammation. Nat Rev Immunol (2016) 16 (8):469-84. doi: 10.1038/nri.2016.62

41. Zhang LK, Wang B, Xin Q, Shang W, Shen S, Xiao G, et al. Quantitative Proteomic Analysis Reveals Unfolded-Protein Response Involved in Severe Fever With Thrombocytopenia Syndrome Virus Infection. J Virol (2019) 93 (10):e00308-19 doi: 10.1128/JVI.00308-19

42. Lundu T, Tsuda Y, Ito R, Shimizu K, Kobayashi S, Yoshii K, et al. Targeting of Severe Fever With Thrombocytopenia Syndrome Virus Structural Proteins to the ERGIC (Endoplasmic Reticulum Golgi Intermediate Compartment) and Golgi Complex. Biomed Res (2018) 39(1):27-38. doi: 10.2220/biomedres.39.27

43. Liu J, Xu M, Tang B, Hu L, Deng F, Wang H, et al. Single-Particle Tracking Reveals the Sequential Entry Process of the Bunyavirus Severe Fever With Thrombocytopenia Syndrome Virus. Small (2019) 15(6):e1803788. doi: $10.1002 / \mathrm{smll} .201803788$

44. Wichgers Schreur PJ, Kortekaas J. Single-Molecule FISH Reveals NonSelective Packaging of Rift Valley Fever Virus Genome Segments. PloS Pathog (2016) 12(8):e1005800. doi: 10.1371/journal.ppat.1005800

45. Jheng JR, Ho JY, Horng JT. ER Stress, Autophagy, and RNA Viruses. Front Microbiol (2014) 5:388. doi: 10.3389/fmicb.2014.00388

46. Hussein IT, Cheng E, Ganaie SS, Werle MJ, Sheema S, Haque A, et al. Autophagic Clearance of Sin Nombre Hantavirus Glycoprotein Gn Promotes Virus Replication in Cells. J Virol (2012) 86(14):7520-9. doi: 10.1128/JVI.07204-11

47. Li W, Cao T, Luo C, Cai J, Zhou X, Xiao X, et al. Crosstalk Between ER Stress, NLRP3 Inflammasome, and Inflammation. Appl Microbiol Biotechnol (2020) 104(14):6129-40. doi: 10.1007/s00253-020-10614-y

48. Sun Y, Jin C, Zhan F, Wang X, Liang M, Zhang Q, et al. Host Cytokine Storm Is Associated With Disease Severity of Severe Fever With Thrombocytopenia Syndrome. J Infect Dis (2012) 206(7):1085-94. doi: 10.1093/infdis/jis452

49. Mills EL, Kelly B, O'Neill LAJ. Mitochondria Are the Powerhouses of Immunity. Nat Immunol (2017) 18(5):488-98. doi: 10.1038/ni.3704

50. Collins LV, Hajizadeh S, Holme E, Jonsson IM, Tarkowski A. Endogenously Oxidized Mitochondrial DNA Induces In Vivo and In Vitro Inflammatory Responses. J leukocyte Biol (2004) 75(6):995-1000. doi: 10.1189/jlb.0703328
51. Moriyama M, Koshiba T, Ichinohe T. Influenza A Virus M2 Protein Triggers Mitochondrial DNA-Mediated Antiviral Immune Responses. Nat Commun (2019) 10(1):4624. doi: 10.1038/s41467-019-12632-5

52. Aarreberg LD, Esser-Nobis K, Driscoll C, Shuvarikov A, Roby JA, Gale MJr. Interleukin-1beta Induces Mtdna Release to Activate Innate Immune Signaling Via Cgas-STING. Mol Cell (2019) 74(4):801-15 e6. doi: 10.1016/ j.molcel.2019.02.038

53. Lai JH, Wang MY, Huang CY, Wu CH, Hung LF, Yang CY, et al. Infection With the Dengue RNA Virus Activates TLR9 Signaling in Human Dendritic Cells. EMBO Rep (2018), 19(8):e46182. doi: 10.15252/embr.201846182

54. Aguirre S, Luthra P, Sanchez-Aparicio MT, Maestre AM, Patel J, Lamothe F, et al. Dengue Virus NS2B Protein Targets cGAS for Degradation and Prevents Mitochondrial DNA Sensing During Infection. Nat Microbiol (2017) 2:17037. doi: 10.1038/nmicrobiol.2017.37

55. Islam MN, Das SR, Emin MT, Wei M, Sun L, Westphalen K, et al. Mitochondrial Transfer From Bone-Marrow-Derived Stromal Cells to Pulmonary Alveoli Protects Against Acute Lung Injury. Nat Med (2012) 18(5):759-65. doi: $10.1038 / \mathrm{nm} .2736$

56. Wang X, Gerdes HH. Transfer of Mitochondria Via Tunneling Nanotubes Rescues Apoptotic PC12 Cells. Cell Death Differ (2015) 22(7):1181-91. doi: 10.1038/cdd.2014.211

57. Sansone P, Savini C, Kurelac I, Chang Q, Amato LB, Strillacci A, et al Packaging and Transfer of Mitochondrial DNA Via Exosomes Regulate Escape From Dormancy in Hormonal Therapy-Resistant Breast Cancer. Proc Natl Acad Sci USA (2017) 114(43):E9066-75. doi: 10.1073/pnas. 1704862114

58. McArthur K, Whitehead LW, Heddleston JM, Li L, Padman BS, Oorschot V, et al. BAK/BAX Macropores Facilitate Mitochondrial Herniation and mtDNA Efflux During Apoptosis. Science (2018) 359(6378):eaao6047. doi: 10.1126/ science.aao6047

59. Dhir A, Dhir S, Borowski LS, Jimenez L, Teitell M, Rotig A, et al. Mitochondrial Double-Stranded RNA Triggers Antiviral Signalling in Humans. Nature (2018) 560(7717):238-42. doi: 10.1038/s41586-018-0363-0

60. Wiatrek DM, Candela ME, Sedmik J, Oppelt J, Keegan LP, O'Connell MA. Activation of Innate Immunity by Mitochondrial dsRNA in Mouse Cells Lacking p53 Protein. RNA (2019) 25(6):713-26. doi: 10.1261/rna.069625.118

61. Li S, Li H, Zhang YL, Xin QL, Guan ZQ, Chen X, et al. Sftsv Infection Induces BAK/BAX-Dependent Mitochondrial DNA Release to Trigger Nlrp3 Inflammasome Activation. Cell Rep (2020) 30(13):4370-85.e7. doi: 10.1016/j.celrep.2020.02.105

62. Qu B, Qi X, Wu X, Liang M, Li C, Cardona CJ, et al. Suppression of the Interferon and NF-kappaB Responses by Severe Fever With Thrombocytopenia Syndrome Virus. J Virol (2012) 86(16):8388-401. doi: 10.1128/JVI.00612-12

63. Sun Q, Jin C, Zhu L, Liang M, Li C, Cardona CJ, et al. Host Responses and Regulation by NFkappaB Signaling in the Liver and Liver Epithelial Cells Infected With A Novel Tick-borne Bunyavirus. Sci Rep (2015) 5:11816. doi: 10.1038/ srep11816

64. Lee JH, Shim YR, Seo W, Kim MH, Choi WM, Kim HH, et al. Mitochondrial Double-Stranded RNA in Exosome Promotes interleukin-17 Production Through Toll-Like Receptor 3 in Alcoholic Liver Injury. Hepatology (2019) 72(2):609-25. doi: 10.1002/hep.31041

65. Song P, Zheng N, Zhang L, Liu Y, Chen T, Bao C, et al. Downregulation of Interferon-beta and Inhibition of TLR3 Expression Are Associated With Fatal Outcome of Severe Fever With Thrombocytopenia Syndrome. Sci Rep (2017) 7(1):6532. doi: 10.1038/s41598-017-06921-6

66. Liu MM, Lei XY, Yu H, Zhang JZ, Yu XJ. Correlation of Cytokine Level With the Severity of Severe Fever With Thrombocytopenia Syndrome. Virol J (2017) 14(1):6. doi: 10.1186/s12985-016-0677-1

67. Sharma D, Kanneganti TD. The Cell Biology of Inflammasomes: Mechanisms of Inflammasome Activation and Regulation. J Cell Biol (2016) 213(6):617-29. doi: 10.1083/jcb.201602089

68. Zhao C, Zhao W. Nlrp3 Inflammasome-A Key Player in Antiviral Responses. Front Immunol (2020) 11:211. doi: 10.3389/fimmu.2020.00211

69. Swanson KV, Deng M, Ting JP. The NLRP3 Inflammasome: Molecular Activation and Regulation to Therapeutics. Nat Rev Immunol (2019) 19 (8):477-89. doi: 10.1038/s41577-019-0165-0 
70. Mangan MSJ, Olhava EJ, Roush WR, Seidel HM, Glick GD, Latz E. Targeting the NLRP3 Inflammasome in Inflammatory Diseases. Nat Rev Drug Discov (2018) 17(9):688. doi: 10.1038/nrd.2018.149

71. Liu Q, Zhang D, Hu D, Zhou X, Zhou Y. The Role of Mitochondria in NLRP3 Inflammasome Activation. Mol Immunol (2018) 103:115-24. doi: 10.1016/j.molimm.2018.09.010

72. Yabal M, Calleja DJ, Simpson DS, Lawlor KE. Stressing Out the Mitochondria: Mechanistic Insights Into NLRP3 Inflammasome Activation. J Leukocyte Biol (2019) 105(2):377-99. doi: 10.1002/JLB.MR0318-124R

73. Zhou R, Yazdi AS, Menu P, Tschopp J. A Role for Mitochondria in NLRP3 Inflammasome Activation. Nature (2011) 469(7329):221-5. doi: 10.1038/nature09663

74. Khalil J, Yamada S, Tsukamoto Y, Abe H, Shimojima M, Kato H, et al. The NonStructural Protein NSs of SFTSV Causes Cytokine Storm Through the HyperActivation of NF-Kappab. Mol Cell Biol (2020) 41(3):e00542-20. doi: 10.1128/ MCB.00542-20

75. Ye W, Lei Y, Yu M, Xu Y, Cao M, Yu L, et al. NLRP3 Inflammasome Is Responsible for Hantavirus Inducing interleukin-1beta in THP-1 Cells. Int J Mol Med (2015) 35(6):1633-40. doi: 10.3892/ijmm.2015.2162

76. Ermler ME, Traylor Z, Patel K, Schattgen SA, Vanaja SK, Fitzgerald KA, et al. Rift Valley Fever Virus Infection Induces Activation of the NLRP3 Inflammasome. Virology (2014) 449:174-80. doi: 10.1016/j.virol.2013.11.015

77. Kalveram B, Lihoradova O, Ikegami T. Nss Protein of Rift Valley Fever Virus Promotes Posttranslational Downregulation of the TFIIH Subunit P62. J Virol (2011) 85(13):6234-43. doi: 10.1128/JVI.02255-10

78. Walter CT, Barr JN. Recent Advances in the Molecular and Cellular Biology of Bunyaviruses. J Gen Virol (2011) 92(Pt 11):2467-84. doi: 10.1099/vir.0.035105-0

79. Moriyama M, Igarashi M, Koshiba T, Irie T, Takada A, Ichinohe T. Two Conserved Amino Acids Within the NSs of Severe Fever With Thrombocytopenia Syndrome Phlebovirus Are Essential for Anti-Interferon Activity. J Virol (2018) 92(19):e00706-18. doi: 10.1128/JVI.00706-18

80. Riley JS, Tait SW. Mitochondrial DNA in Inflammation and Immunity. EMBO Rep (2020) 21(4):e49799. doi: 10.15252/embr.201949799

81. Jabir MS, Hopkins L, Ritchie ND, Ullah I, Bayes HK, Li D, et al. Mitochondrial Damage Contributes to Pseudomonas Aeruginosa Activation of the Inflammasome and Is Downregulated by Autophagy. Autophagy (2015) 11(1):166-82. doi: 10.4161/15548627.2014.981915

82. Sun L, Wu J, Du F, Chen X, Chen ZJ. Cyclic GMP-AMP Synthase is a Cytosolic DNA Sensor That Activates the Type I Interferon Pathway. Science (2013) 339(6121):786-91. doi: 10.1126/science.1232458

83. Gao D, Wu J, Wu YT, Du F, Aroh C, Yan N, et al. Cyclic GMP-AMP Synthase Is an Innate Immune Sensor of HIV and Other Retroviruses. Science (2013) 341(6148):903-6. doi: 10.1126/science.1240933

84. Dobbs N, Burnaevskiy N, Chen D, Gonugunta VK, Alto NM, Yan N. Sting Activation by Translocation From the ER Is Associated With Infection and Autoinflammatory Disease. Cell Host Microbe (2015) 18(2):157-68. doi: 10.1016/j.chom.2015.07.001

85. Kerur N, Veettil MV, Sharma-Walia N, Bottero V, Sadagopan S, Otageri P, et al. IFI16 Acts as a Nuclear Pathogen Sensor to Induce the Inflammasome in Response to Kaposi Sarcoma-Associated Herpesvirus Infection. Cell Host Microbe (2011) 9(5):363-75. doi: 10.1016/j.chom.2011.04.008

86. Liu H, Zhang H, Wu X, Ma D, Wu J, Wang L, et al. Nuclear cGAS Suppresses DNA Repair and Promotes Tumorigenesis. Nature (2018) 563(7729):131-6. doi: 10.1038/s41586-018-0629-6

87. Carpenter S, Aiello D, Atianand MK, Ricci EP, Gandhi P, Hall LL, et al. A Long Noncoding RNA Mediates Both Activation and Repression of Immune Response Genes. Science (2013) 341(6147):789-92. doi: 10.1126/science.1240925

88. Villarroya-Beltri C, Gutierrez-Vazquez C, Sanchez-Cabo F, Perez-Hernandez D, Vazquez J, Martin-Cofreces N, et al. Sumoylated hnRNPA2B1 Controls the
Sorting of miRNAs Into Exosomes Through Binding to Specific Motifs. Nat Commun (2013) 4:2980. doi: 10.1038/ncomms3980

89. Alarcon CR, Goodarzi H, Lee H, Liu X, Tavazoie S, Tavazoie SF. Hnrnpa2b1 Is a Mediator of $\mathrm{M}(6) \mathrm{a}-$ Dependent Nuclear Rna Processing Events. Cell (2015) 162(6):1299-308. doi: 10.1016/j.cell.2015.08.011

90. Wang L, Wen M, Cao X. Nuclear hnRNPA2B1 Initiates and Amplifies the Innate Immune Response to DNA Viruses. Science (2019) 365(6454):eaav0758. doi: 10.1126/science.aav0758

91. Konno H, Konno K, Barber GN. Cyclic Dinucleotides Trigger ULK1 (ATG1) Phosphorylation of STING to Prevent Sustained Innate Immune Signaling. Cell (2013) 155(3):688-98. doi: 10.1016/j.cell.2013.09.049

92. Tanaka Y, Chen ZJ. STING Specifies IRF3 Phosphorylation by TBK1 in the Cytosolic DNA Signaling Pathway. Sci Signaling (2012) 5(214):ra20. doi: 10.1126/scisignal.2002521

93. Cao L, Liu S, Li Y, Yang G, Luo Y, Li S, et al. The Nuclear Matrix Protein Safa Surveils Viral RNA and Facilitates Immunity by Activating Antiviral Enhancers and Super-Enhancers. Cell Host Microbe (2019) 26(3):369-84 e8. doi: 10.1016/j.chom.2019.08.010

94. Kerr JF, Wyllie AH, Currie AR. Apoptosis: A Basic Biological Phenomenon With Wide-Ranging Implications in Tissue Kinetics. Br J Cancer (1972) 26 (4):239-57. doi: 10.1038/bjc.1972.33

95. Carneiro BA, El-Deiry WS. Targeting Apoptosis in Cancer Therapy. Nat Rev Clin Oncol (2020) 17(7):395-417. doi: 10.1038/s41571-020-0341-y

96. Bock FJ, Tait SWG. Mitochondria as Multifaceted Regulators of Cell Death. Nat Rev Mol Cell Biol (2020) 21(2):85-100. doi: 10.1038/s41580-019-0173-8

97. Mehta MM, Weinberg SE, Chandel NS. Mitochondrial Control of Immunity: Beyond ATP. Nat Rev Immunol (2017) 17(10):608-20. doi: $10.1038 /$ nri.2017.66

98. Green DR. The Coming Decade of Cell Death Research: Five Riddles. Cell (2019) 177(5):1094-107. doi: 10.1016/j.cell.2019.04.024

99. Wang Y, Ning X, Gao P, Wu S, Sha M, Lv M, et al. Inflammasome Activation Triggers Caspase-1-Mediated Cleavage of cGAS to Regulate Responses to DNA Virus Infection. Immunity (2017) 46(3):393-404. doi: 10.1016/ j.immuni.2017.02.011

100. Ning X, Wang Y, Jing M, Sha M, Lv M, Gao P, et al. Apoptotic Caspases Suppress Type I Interferon Production Via the Cleavage of Cgas, MAVS, and IRF3. Mol Cell (2019) 74(1):19-31.e7. doi: 10.1016/j.molcel. 2019.02.013

101. White MJ, McArthur K, Metcalf D, Lane RM, Cambier JC, Herold MJ, et al. Apoptotic Caspases Suppress mtDNA-Induced STING-Mediated Type I IFN Production. Cell (2014) 159(7):1549-62. doi: 10.1016/j.cell. 2014.11.036

102. Zhou X, Jiang W, Liu Z, Liu S, Liang X. Virus Infection and Death ReceptorMediated Apoptosis. Viruses (2017) 9(11):316. doi: 10.3390/v9110316

103. Kvansakul M, Caria S, Hinds MG. The Bcl-2 Family in Host-Virus Interactions. Viruses (2017) 9(10):290. doi: 10.3390/v9100290

Conflict of Interest: The authors declare that the research was conducted in the absence of any commercial or financial relationships that could be construed as a potential conflict of interest.

Copyright (C) 2021 Zhou and Yu. This is an open-access article distributed under the terms of the Creative Commons Attribution License (CC BY). The use, distribution or reproduction in other forums is permitted, provided the original author(s) and the copyright owner(s) are credited and that the original publication in this journal is cited, in accordance with accepted academic practice. No use, distribution or reproduction is permitted which does not comply with these terms. 